\title{
Optical and Structural Properties of GaAs/GaInP Quantum Wells Grown by Chemical Beam Epitaxy
}

\author{
M. R. Martins ${ }^{1}$, J. B. B. Oliveira ${ }^{1}$, A. Tabata ${ }^{1}$, E. Laureto ${ }^{2}$, \\ J. Bettini ${ }^{3}$, E. A. Meneses ${ }^{2}$, and M. M. G. Carvalho ${ }^{2}$ \\ ${ }^{1}$ Departamento de Física, Universidade Estadual Paulista - UNESP, Campus de Bauru, São Paulo, Brazil \\ ${ }^{2}$ Instituto de Física “ Gleb Wathagin”, UNICAMP, Campinas, São Paulo, Brazil \\ ${ }^{3}$ Laboratório Nacional de Luz Sincroton - LNLS, Campinas, São Paulo, Brazil
}

Received on 31 March, 2003

\begin{abstract}
In this work we investigated the optical and structural properties of GaAs/GaInP quantum wells (QW) grown by Chemical Beam Epitaxy (CBE). The samples were characterized by photoluminescence (PL), photoluminescence excitation (PLE) and transmission electron microscopy (TEM). Simulations of the quantum well potential profiles, using the Van De Walle-Martin model, supplemented by our experimental results, allowed us to associate the interface properties with the growth procedures. We concluded that a thin GaP layer grown at the interface improves its quality and also that the observed broad emission band in the PL spectrum is related to quaternary $\mathrm{Ga}_{1-x} \mathrm{In}_{x} \mathrm{As}_{1-y} \mathrm{P}_{y}$.
\end{abstract}

\section{Introduction}

GaInP alloy is a strong candidate to substitute GaA1As alloy in the manufacture of optoeletronic devices mainly due to the low reactivity with oxygen, minor carrier recombination speed at the surface and low presence of D-X deep centers. However, there are many obstacles in the growth of these heterostructures which have relatively little interface quality. The main phenomena that influence the GaAs/GaInP QW sample quality are: memory effect resulting in a spurious incorporation of group $\mathrm{V}$ elements (As or P), In segregation in the interfaces and the intricate As-P (or P-As) exchange mechanism during the growth. Due to these phenomena, it is difficult to grow abrupt interfaces and to avoid the formation of a non-intentional ternary and/or quaternary layer at the interface [1,2]. In this study, we investigated the influence of the growth conditions for $\mathrm{GaAs} / \mathrm{Ga}_{0.51} \mathrm{In}_{0.49} \mathrm{P}$ multiple quantum well. The samples were grown at temperature of $500^{\circ} \mathrm{C}$ using $\mathrm{CBE}$. The GaInP and $\mathrm{GaAs}$ layers are $300 \AA$ and $50 \AA$ wide, respectively, and have been grown on alternately on (100) GaAs substrates. The three studied samples are representative of a set of samples that were grown on the same condition. Our analysis shows that to improve the interfaces quality it is important to perform a surface passivation by means of $\mathrm{P}$ and As flows exposition, and that the first interface ( $\mathrm{GaAs}$ grown on $\mathrm{GaInP}$ ) determines the morphologic quality of the samples. For these reasons, we have adopted different growth procedures for the first interface, while in the second (GaInP grown on GaAs) no modification was adopted. Concerning the mentioned three samples, the procedures in the interfaces growth were as follow: a) $\mathrm{P}$ flow during $15 \mathrm{~s}$ for the sample A; b) P flow during $15 \mathrm{~s}$ followed by As flow for $30 \mathrm{~s}$, for the sample B; c) P flow for $15 \mathrm{~s}$, followed by three GaP monolayer (ML) and exposition to As flow during 30s, for the sample C. The PL and PLE measurements were obtained by using a T64000 JobinYvon system with the samples in a closed-circuit He cryostat. The theoretical simulations were done using the Van de Walle-Martin model [3,4].

\section{Results and Discussions}

Figure 1 shows the PL and PLE spectra for samples A, B and $\mathrm{C}$. Table 1 lists the experimental values of full width at the half maximum (FWHM) of the PL fundamental transition, Stokes Shift (SS) and the fundamental absorption energy value $\left(\mathrm{E}_{1}-\mathrm{HH}_{1}\right)$ for analysed samples. In the PL spectra of the samples A and C, we clearly observed the excitonic emission associated to the QW confinement. The PL spectrum of the sample B presents a broad emission band at $1.474 \mathrm{eV}$. The nature of this band will be analysed below. In the PLE spectra of samples A and C we observed peaks related to the transitions between electrons and both light $\left(\mathrm{E}_{1}-\mathrm{LH}_{1}\right)$ and heavy $\left(\mathrm{E}_{1}-\mathrm{HH}_{1}\right)$ holes, while in the spectrum of sample B these peaks are not so clear.

These results show a clear difference between sample B and the others (A and $\mathrm{C}$ ). Also indicate that the interface quality is worse for this sample. This fact is evidenced from the FWHM and SS data. Low values were found for samples $\mathrm{A}$ and $\mathrm{C}$, while the values obtained for the sample $\mathrm{B}$ are very high. The values of FWHM and SS for sample A are higher than those of the sample $\mathrm{C}$, suggesting us that its interfaces are flat. This result is coherent with the fact that in the growth of sample A the As-P exchange was intense. The best QW interfaces were obtained in the sample $\mathrm{C}$, 


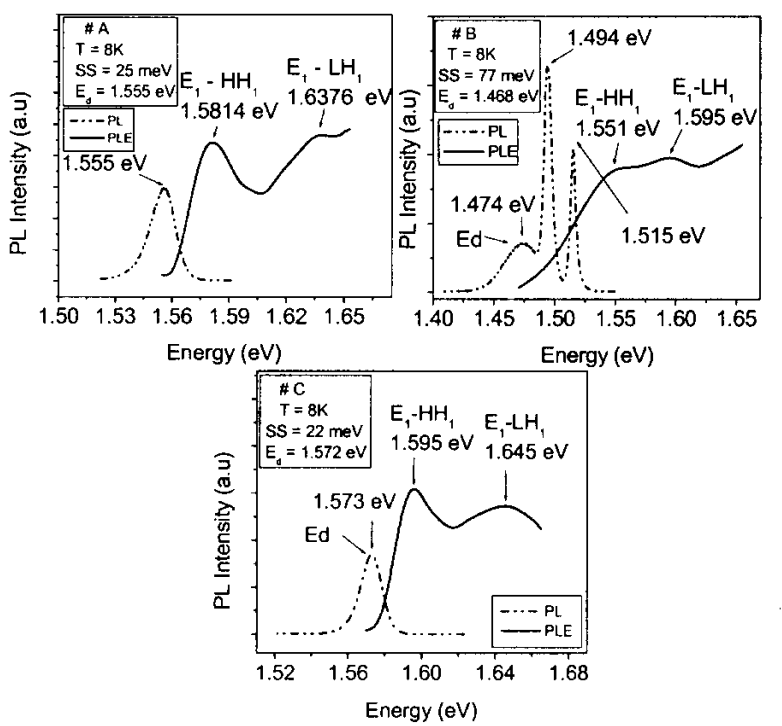

Figure 1. PL (dashed line) and PLE (continuous line) for the samples A, B and C.

TABLE 1: Experimenta; FWHM $\mathrm{PL}_{\mathrm{L}}$, SS e $\mathrm{E}_{1}-\mathrm{HH}_{1}$ values for samples $\mathrm{A}, \mathrm{B}$ and $\mathrm{C}$.

\begin{tabular}{cccc}
\hline Samples & $\mathrm{FWHM}_{P L}(\mathrm{meV})$ & $\mathrm{SS}(\mathrm{meV})$ & $\mathrm{E}_{1}-\mathrm{HH}_{1}(\mathrm{eV})$ \\
\hline $\mathrm{A}$ & 15 & 25 & 1.581 \\
\hline $\mathrm{B}$ & 35 & 77 & 1.551 \\
\hline $\mathrm{C}$ & 13 & 22 & 1.595 \\
\hline
\end{tabular}

in which three GaP ML have been grown at the interface, after the exposition to $\mathrm{P}$ flow and before the exposition to As flow. The GaP inter layer acts in order to prevent the In segregation and the P-As exchange. These optical measurements are in agreement with theoretical simulations results, using the Van de Walle-Martin model [3,4]. For the ideal QW with abrupt interface the calculated $\mathrm{E}_{1}-\mathrm{HH}_{1}$ transition energy is $1.589 \mathrm{eV}$. In these simulations, if we take into account the presence of narrow InGaAs ternary or $\mathrm{Ga}_{1-x} \mathrm{In}_{x} \mathrm{As}_{y} \mathrm{P}_{1-y}$ quaternary layers at the interface, a drastic shift of the fundamental QW optical transition to low energies side occurs, as observed in the sample B. It suggests that these spurious layers at the interfaces could be formed on this sample. On the other hand, simulation considering a GaP layer at the interfaces results in a shift to high energies. As we have observed in the optical results. Fig. 2 displays the TEM images for analysed samples. Observating the TEM images it is possible to note that the roughness in the interface is more evident in the sample A, as well as detected from the optical measurements and related to the As-P exchange. It is important to note that the exposition of the GaInP layer to the As flow during 30s contributes to smooth the surface. It decreases the roughness level as it can be noticed in the TEM images of the samples B and C. In addition, we can speculated the origin of some features of the TEM images. The dark region in the TEM image of sample $\mathrm{B}$ is, probably, associated to $\mathrm{Ga}_{1-x} \operatorname{In}_{x} \mathrm{As}_{y} \mathrm{P}_{1-y}$ with high concentrations of In and As, while the clear region in TEM image of sample $\mathrm{C}$ is also associated to the quaternary, but
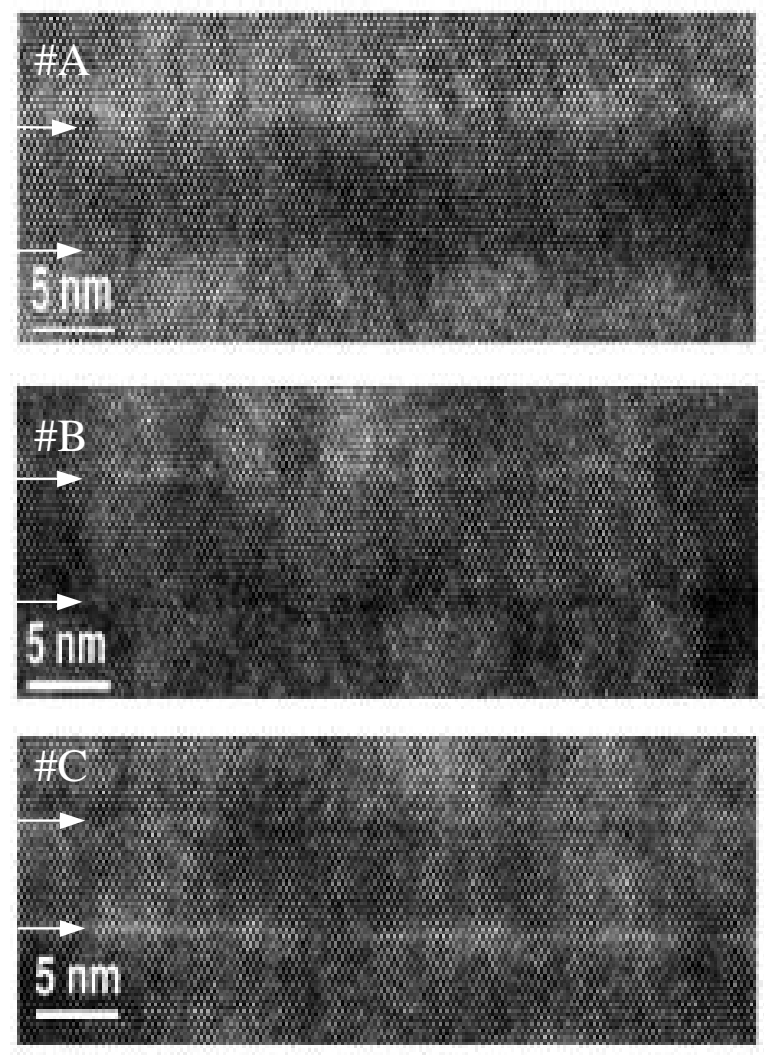

Figure 2. TEM images for the samples A, B and C, the arrows indicate the interface.

with low concentrations of In and As. These assumptions are in accordance with the optical measurements, mainly for the sample B in which we found indication (see bellow) that the broad band in the low energy side of the PL spectrum is associated to the non intentional grown layer at the interfaces. These results show that for the sample B, obtained by the exposition of the surfaces to the As and $\mathrm{P}$ flows, despite the low roughness, as compared to the sample A, the spurious layers acts in the direction to degrade the general quality of the sample.

In order to investigate the nature of the broad band emission at low energy, we carried out PL measurements for sample B with different excitation energies. The spectra are shown in Fig. 3. When the carriers are photocreated directly in the well (sample excited with $1.70 \mathrm{eV}$ ) the broad band intensity is relatively small, if compared with the intensity of the emissions from the GaAs $(1.515 \mathrm{eV})$ and carbon $(1.494$ $\mathrm{eV})$. On the other hand, when the carriers are created in the barrier (sample excited with $2.51 \mathrm{eV}$ ) the intensity of this emission increases drastically and different peaks appear. In this way, we can conclude that the broad band emission has the origin on part of the barrier, more probably, on the $\mathrm{Ga}_{1-x} \mathrm{In}_{x} \mathrm{As}_{y} \mathrm{P}_{1-y}$ quaternary formed by As incorporation by the GaInP barrier. 


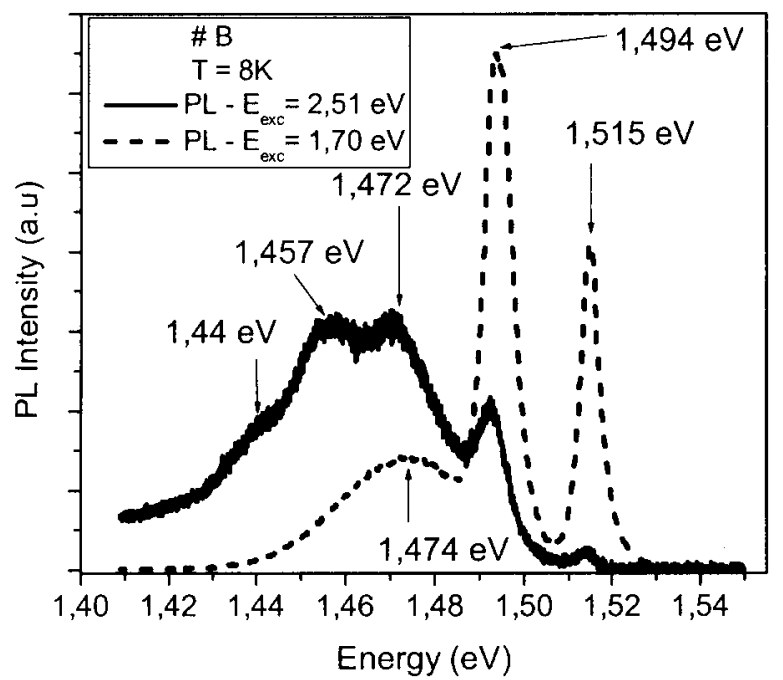

Figure 3. PL spectra of the sample B using different excitation energies.

\section{Conclusions}

In summary, our PL, PLE and TEM experimental results have shown that the exposition of the surfaces to the $\mathrm{P}$ and As flow are important to smoothing the GaAs/GaInP QW interfaces, mainly the one formed by the deposition of GaAs on GaInP. Also we have observed that better interface is achieved by the growth of a narrow GaP inter layer prob- ably because it prevents the In incorporation and facilitates the As-P exchange process, preventing the formation of spurious layers at the interfaces. Theoretical simulations, TEM images and PL measurements using different excitation energy have shown indications that the broad band emission at $\sim 1.47 \mathrm{eV}$ is associated to spurious $\mathrm{Ga}_{1-x} \mathrm{In}_{x} \mathrm{As}_{y} \mathrm{P}_{1-y}$ quaternary layer. The exposition of the surfaces to the As and $P$ flows without a thin GaP layer results in a smooth surface but it favors the formation of non intentional layers that compromise the interfaces quality. Finally, our results allowed us to establish a coherent relation between the structural and optical properties of the samples with the adopted growth procedures at the interfaces.

\section{Acknowledgement}

The authors gratefully acknowledge the financial support from FAPESP, CNPq and FUNDUNESP.

\section{References}

[1] T.K. Sharma, B.M. Arora, M.R. Gokhale and S. Rajgopalan J. Crystal Growth 221, 509 (2000).

[2] T.K. Sharma, M.R. Gokhale and B.M. Arora, J. Crystal Growth 213, 241 (2000).

[3] C.G. Van de Walle and R. M. Martin, Phys. Rev., B34 5621 (1986); B35 8154 (1987).

[4] C. Lamberti, Comp. Phys. Commun., 93, 82 (1996). 